\title{
Acknowledgment to Reviewers of Atoms in 2021
}

\section{Atoms Editorial Office}

Citation: Atoms Editorial Office. Acknowledgment to Reviewers of Atoms in 2021. Atoms 2022, 10, 15. https://doi.org/10.3390/ atoms10010015

Published: 27 January 2022

Publisher's Note: MDPI stays neutral with regard to jurisdictional claims in published maps and institutional affiliations.

Copyright: (c) 2022 by the authors. Licensee MDPI, Basel, Switzerland. This article is an open access article distributed under the terms and conditions of the Creative Commons Attribution (CC BY) license (http://creativecommons.org/licenses/by/4.0/).

MDPI AG, St. Alban-Anlage 66, 4052 Basel, Switzerland

Rigorous peer-reviews are the basis of high-quality academic publishing. Thanks to the great efforts of our reviewers, Atoms was able to maintain its standards for the high quality of its published papers. Thanks to the contribution of our reviewers, in 2021, the median time to first decision was 18 days, and the median time to publication was 38.5 days. The editors would like to extend their gratitude and recognition to the following reviewers for their precious time and dedication, regardless of whether the papers they reviewed were finally published.

Agueny, Hicham

Alijah, Alexander

Amaro, Pedro

Amelio, Ivan

Andreev, Oleg Yu.

Armstrong, Gregory

Aubin, Seth

Bakr, Mahmoud

Bartschat, Klaus

Belmonte, Maria Teresa

Ben Nessib, Nabil

Benda, Jakub

Benis, Emmanouil P.

Bentz, Wolfgang

Bertoldi, Andrea

Borghesani, Armando Francesco

Borschevsky, Anastasia

Braams, Bastiaan J.

Brage, Tomas

Branca, Antonio

Bray, Igor

Bredehöft, Jan

Bromley, Michael

Brown, Greg

Buckman, Stephen

Camacho-Guardian, Arturo

Camiola, Vito Dario

Cembranos, Jose

Chai, Shijie

Chiatti, Leonardo

Christova, Magdalena

Clougherty, Dennis P.

Colonna, Gianpiero

Constantin, Florin Lucian

Kar, Sabyasachi
Del Zanna, Giulio

Demura, Alexander

Devdariani, Alexander

Dimitrijevic, Milan S.

Domi, Alba

Drake, Gordon

Duca, Lucia

Dunningham, Jacob A.

Fabrikant, Ilya

Fontes, Christopher J.

Foord, Mark

Froese Fischer, Charlotte

Fursa, Dmitry

Garrido Alzar, Carlos L.

Gautam, Siddharth

Ginges, Jacinda

Gryzlova, Elena V.

Gumberidze, Alexandre

Gupta, Dhanoj

Haga, Taiki

Haine, Simon

Han, Jianing

Hansen, Stephanie B.

Hasuo, Masahiro

Hibbert, Alan

Hinojosa, Guillermo

Hoffmann, Andreas

Hutton, Roger

Ibrahim, Abdel-Baset M. A.

Jachymski, Krzysztof

Janíček, Petr

Jaroszinski, Dino

Kabachnik, N. M.

Kajita, Masatoshi

Parish, Meera 
Karwasz, Grzegorz

Karwowski, Boleslaw

Kato, Daiji

Khakoo, Murtadha

Kirchner, Tom

Koike, Fumihiro

Konobeev, Alexander

Koubiti, Mohammed

Kovachy, Tim

Kowalczyk, Tim

Kozlov, Mikhail

Kramida, Alexander

Kukushkin, Alexander B.

Kumarakrishnan, A.

Lai, Yu Hang

Machacek, Joshua

Maksimov, Dmitrii N.

Manson, Steven

Marinkovic, Bratislav

Maslov, Mikhail M

Mendonça, José Tito

Milosavljevic, Aleksandar

Mohallem, José R.

Mohan, Man

Moreno-Díaz, Cristina

Mozejko, Pawel

Mukherjee, Koushik

Nagirner, Dmitrij I.

Narducci, Francesco

Narits, Alexander A.

Navarrete, Francisco Oscar

Norito, Ishikawa

Ojeda-May, Pedro

Okon, Ituen B.

Okopinska, Anna

Oller, Jose Antonio

Ončák, Milan

O'Sullivan, Gerard

Otranto, Sebastian

Ouvry, Stephane

Palazzatti, Federico

Pandey, Mukesh Kumar

Parente, Fernando
Peña Ardila, Luis

Piwiński, Mariusz

Politano, Grazia Giuseppina

Poparić, Goran

Poveda, Luis A.

Puchalski, Mariusz

Pullman, David P.

Raineri, Mónica M.

Reid, David D.

Richaud, Andrea

Rittenhouse, Seth T.

Robb, Gordon

Rodrigues, Sérgio

Romero-Rochin, Victor

Ruiz Ruiz, María Belén

Russomanno, Angelo

Sakan, Nenad

Salam, Akbar

Samson, E. Carlo

Santos, José Paulo

Scheier, Paul

Schippers, Stefan

Schowalter, Marco

Schultze, Martin

Shapiro, David

Slama, Sebastian

Stambulchik, Evgeny

Stefani, Giovanni L.

Suzuki, Jun

Syrocki, Łukasz

Takahashi, Kaito

Tantardini, Christian

Tokesi, Karoly

Tomza, Michał

Träbert, Elmar

Van Hoof, Peter

Villarreal, Carlos

Volosniev, Artem

Vovrosh, Jamie

Weyrauch, Michael

Whelan, Colm

Wolf, Robert C.

Zammit, Mark C. 